\title{
Hair Styling with Physics
}

\section{Experiments show that hair-like bundles form different shapes depending on the speed at which they are dried.}

\section{By Michael Schirber}

S ameh Tawfick and his teammates came across a strange occurrence a few years ago while studying how carbon nanotubes self-assemble in liquid: the tubes formed different structures depending on how fast they dried. Curious to know the reason for this drying dependence, the team-based at the University of Illinois at Urbana-Champaign-began a series of experiments on micrometer-thick carbon fibers, or "hairs." In their latest study, they monitor how the speed at which liquid drains from a bundle of hairs determines the bundle's shape when dry [1]. Untangling the role that hydrodynamics plays in fiber morphology could allow the design of smart fibers that can be controlled with wetting.

Normally, clothes and other fiber-containing materials dry slowly, taking final forms based on a competition between fiber elasticity-which resists deformation-and capillary forces-which tend to shrink the fibers toward each other. However, as Tawfick and others have recently documented, rapid drying can alter the final fiber configuration.

To investigate this effect, Tawfick and colleagues assembled

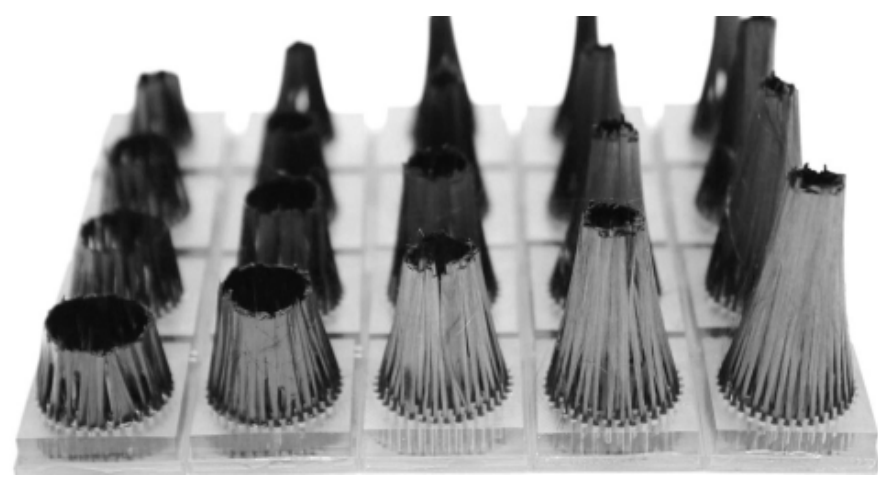

Credit: J. Ha and Y. S. Kim/University of Illinois at Urbana-Champaign.
Credit: J. Ha and Y. S. Kim/University of Illinois at Urbana-Champaign

hair bundles in configurations that looked like hollow paint brushes. The bundles were then submerged in a liquid bath and removed. Slow removal (slow drying) produced a rigid wall of hairs in an open ring shape, whereas quick removal (fast drying) caused the bundles to collapse into closed points. The team explained this closure as being caused by a liquid column that forms in the hollow center and pulls the bundle closed through surface tension. By contrast, slow drying gives time for liquid to drain from the center, so no column forms. This model may help researchers understand the way wet fibers trap air in otter fur and duck feathers, which could lead to bio-inspired materials.

Michael Schirber is a Corresponding Editor for Physics based in Lyon, France.

\section{REFERENCES}

1. J. Ha et al., "Hydrodynamic elastocapillary morphing of hair bundles," Phys. Rev. Lett. 125, 254503 (2020). 